\title{
FBXW7 mediates high glucose-induced SREBP-1 expression in renal tubular cells of diabetic nephropathy under PI3K/Akt pathway regulation
}

\author{
LISHA LI $^{1 *}$, JUXIANG YANG ${ }^{2 *}$, FAN LI $^{3,4}$, FAN GAO $^{3,4}$, LIN ZHU $^{5}$ and $\mathrm{JUN} \mathrm{HAO}^{3,4}$ \\ ${ }^{1}$ Department of Pathology, Cangzhou Hospital of Integrated TCM-WM, Cangzhou, Hebei 061001; \\ ${ }^{2}$ The Office of Basic Medical College and ${ }^{3}$ Department of Pathology, Hebei Medical University, Shijiazhuang, Hebei 050017; \\ ${ }^{4}$ Hebei Key Laboratory of Kidney Diseases, Shijiazhuang, Hebei 050017; ${ }^{5}$ Department of Electromyogram, \\ The Third Hospital of Hebei Medical University, Shijiazhuang, Hebei 050001, P.R. China
}

Received July 14, 2020; Accepted December 15, 2020

DOI: $10.3892 / \mathrm{mmr} .2021 .11872$

\begin{abstract}
Diabetic nephropathy (DN) is a severe complication of diabetes mellitus and lipid metabolism abnormality serves a key role in the pathogenesis of DN. Sterol regulatory element-binding protein 1 (SREBP-1) overexpression mediates aberrant lipid accumulation in renal tubular cells of DN. However, the exact mechanism involved in increased SREBP-1 has not been fully elucidated. The aim of the present study was to explore the mechanism involved in SREBP-1 upregulation. Diabetic mice and high glucose-cultured HKC cells were chosen to detect the expression of FBXW7 and SREBP-1 using immunohistochemistry, western blotting and PCR. The present study demonstrated that F-box and WD repeat domain containing 7 (FBXW7) expression was decreased in renal tubular cells of diabetic mice. Moreover, the co-expression of FBXW7 and SREBP-1 was observed in renal tubular cells, but not in the glomeruli. High glucose-induced the downregulation of FBXW7 expression in in vitro cultured HKC cells, which was accompanied by SREBP-1 upregulation. In addition, overexpression of FBXW7 in HKC cells led to SREBP-1 downregulation. By contrast, knockdown of FBXW7 caused SREBP-1 upregulation in HKC cells. It was
\end{abstract}

Correspondence to: Dr Lin Zhu, Department of Electromyogram, The Third Hospital of Hebei Medical University, 139 Ziqiang Road, Shijiazhuang, Hebei 050001, P.R. China

E-mail: zhulin_930@163.com

Professor Jun Hao, Department of Pathology, Hebei Medical University, 361 Zhongshan East Road, Shijiazhuang, Hebei 050017, P.R. China

E-mail: haojun2004@hotmail.com

${ }^{*}$ Contributed equally

Key words: F-box and WD repeat domain containing 7, high glucose, sterol regulatory element-binding protein 1, PI3K/Akt pathway, diabetic nephropathy, renal tubular cells found that the PI3K/Akt signaling pathway was activated in high glucose-stimulated HKC cells, and inhibition of PI3K/Akt pathway using LY294002 increased FBXW7 expression and decreased SREBP-1 expression. Taken together, the present results suggested that FBXW7 mediated high glucose-induced SREBP-1 expression in renal tubular cells of DN, under the regulation of the PI3K/Akt signaling pathway.

\section{Introduction}

Diabetic nephropathy (DN) is a type of severe complication of diabetes mellitus (DM) and is the main cause of chronic kidney disease (1). Various of mechanism are reported to be involved in the pathogenesis and development of DN, such as hyperglycemia, oxidative stress (2), mitochondrial dysfunction (3), endoplasmic reticulum stress, chronic inflammation and abnormal autophagy (4). Recently, accumulating evidence has revealed that lipid metabolism abnormality serves an important role in the pathogenesis of DN (5). In both diabetic animals and patients, renal mesangial cells, renal tubular cells and podocytes experience lipid accumulation, which induces extracellular matrix (ECM) deposition, including fibronectin, collagen I, collagen 3 mainly located in renal tubular interstitium and collagen 4 mainly located in renal glomeruli, and fibrosis $(6,7)$.

Sterol regulatory element-binding protein 1 (SREBP-1) is a key transcription factor of lipid metabolism, especially fatty acid synthesis (8). In a previous study, SREBP-1 was proven to regulate the lipid metabolism abnormality of DN in renal tubular cells (9). Hyperglycemia increases SREBP-1 expression, leading to fatty acid synthase, acetol-CoA carboxylase upregulation and triglyceride augment in renal tubular cells (9). However, the exact mechanism involved in hyperglycemia-induced increased SREBP-1 is yet to be fully elucidated.

F-box and WD repeat domain containing 7 (FBXW7) is a type of E3 ubiquitin ligase that belongs to the SCF E3 ubiquitin ligase family, and has multiple downstream targets, such as c-Myc, MCL1 apoptosis regulator, BCL2 family member, c-Jun, Notch1 and cyclin E (10). Moreover, FBXW7 
is a multifunctional protein and regulates cell proliferation, apoptosis, survival and aging (11). FBXW7 also serves a role in affecting tumor growth in neoplasm genesis and progression, acting as tumor suppressor gene (12). Increasing evidence has revealed that SREBP-1 is another downstream target of FBXW7 (13). Therefore, we hypothesized that FBXW7 may mediate high glucose-induced SREBP-1 upregulation in renal tubular cells.

In the present study, diabetic mice and a human renal tubular cell line (HKC) were chosen to detect the expression levels of FBXW7 and SREBP-1. Furthermore, FBXW7 expression was upregulated or downregulated using expression plasmid or short hairpin (sh)RNA plasmid to investigate the direct relationship between FBXW7 and SREBP-1. Considering that the PI3K/Akt pathway is the critical cell signaling pathway affecting DN (14), the potential role of the PI3K/Akt pathway on FBXW7 expression in HKC cells was determined.

\section{Materials and methods}

Reagents and materials. The antibody against SREBP-1 (cat. no. sc-13551, dilution 1:200 for immunohistochemistry, immunocytochemistry and immunofluorescence; dilution 1:1,000 for western blotting) was purchased from Santa Cruz Biotechnology, Inc. The antibody against FBXW7 was obtained from Aviva Systems Biology Corp (cat. no. ARP47419_P050, dilution 1:200 for immunohistochemistry, immunocytochemistry and immunofluorescence, 1:1,000 for western blotting). The antibodies against phosphorylated (p)-Akt (Ser 473; cat. no. 4060, dilution 1:1,000 for western blotting) and Akt (cat. no. 4691, dilution 1:1,000 for western blotting) were purchased from Cell Signaling Technology, Inc. The antibody against nestin was bought from Abcam (cat. no. ab221660, dilution 1:200 for immunofluorescence). The antibody against $\beta$-actin was purchased from ABclonal Biotechnology Co., Ltd. (cat. no. AC026, dilution 1:1,000 for western blotting). Streptozotocin (STZ) was obtained from Sigma-Aldrich (Merck KGaA), while LY294002 was purchased from MedChemExpress. Lipofectamine ${ }^{\circledR} 2000$ was purchased from Thermo Fisher Scientific, Inc. and TRIzol ${ }^{\circledR}$ from Invitrogen (Thermo Fisher Scientific, Inc.). The PrimeScript ${ }^{\mathrm{TM}}$ reverse transcription (RT) reagent kit with gDNA Eraser and SYBR ${ }^{\circledR}$ Premix Ex Taq ${ }^{\mathrm{TM}}$ II (Tli RNaseH Plus) were obtained from Takara Bio, Inc. An immunohistochemistry kit was purchased from Beijing Zhongshan Golden Bridge Technology Co., Ltd. DyLight 488 or 594-labelled goat secondary antibodies were purchased from KPL, Inc. The FBXW7 expression plasmid (pcDNA3.0-FBXW7) was bought from Sino Biological. The pGenesil-1 plasmid (Wuhan An Di Jing Sai Bio-Technology Co., Ltd.) for shRNA plasmid construction was stored in the authors' lab. Glucose and mannitol were bought from Beijing Solarbio Science \& Technology Co., Ltd.

Diabetic mice. A total of 20 male CD1 mice aged six weeks (weight 20-25 g) were purchased from Beijing Vital River Laboratory Animal Technology Co., Ltd. and were fed with free access to water and food. All animal experiments were according to the rules of Institutional Animal Care and Use Committee of the Third Hospital of Hebei Medical University (permit no. Guo A 2017-019-1; period, 2017-2020). All animals were housed with 12 -h light/dark cycle, $20 \pm 3^{\circ} \mathrm{C}$ and $35-55 \%$ humidity. A total of 10 mice were intraperitoneally injected with STZ (150 mg/kg body weight) to establish diabetic models (15) and the corresponding 10 control mice were injected with equivalent volume sodium citrate solution ( $1.29 \%$ sodium citrate, $1.18 \%$ citric acid). Then, 3 day after the injection, mice with measured blood glucose levels of $>16.7 \mathrm{mmol} / \mathrm{l}$ were regarded as successfully established models. All animals were fed for 16 weeks and then sacrificed. Mice were euthanized by exsanguination after deep $4 \%$ isoflurane anesthesia, and death was confirmed by the lack of reflexes of paw withdrawal and eye blink. Mice with a lack of breathing and heartbeat were considered dead, and the renal cortex was collected for the subsequent experiments.

Cell culture and grouping. A human renal tubular cell line (HKC) was kindly provided by Professor Chen Xiang-Mei, Division of Nephropathy, 301 Hospital (Beijing, China). The HKC cell line was originally established by Racusen et al (16) who isolated human renal tubule epithelial cells and exposed them in culture to a hybrid immortalizing virus, adenovirus 12-SV40. The cells were cultured as described previously (9) and randomly divided into three groups to examine the effect of high glucose: Normal glucose group $(5.5 \mathrm{mmol} / \mathrm{l}$ glucose; N), high glucose group (30 mmol/l glucose; $\mathrm{H})$ and mannitol group (30 mmol/l mannitol; M). After the indicated times (at $37^{\circ} \mathrm{C}$ for 36,48 and $72 \mathrm{~h}$ ), the various assays were performed.

To investigate the effect of FBXW7 overexpression on SREBP-1 expression, HKC cells were divided into untransfected group, pcDNA3.0 group and pcDNA3.0-FBXW7 group. Moreover, to determine the effect of FBXW7 knockdown on SREBP-1 expression, HKC cells were divided into untransfected group, pGenesil-1 group, pGenesil-1-FBXW7-1 group and pGenesil-1-FBXW7-2 group. To evaluate the role of the PI3K/Akt pathway in regulating FBXW7 expression, $\mathrm{HKC}$ cells were divided into $\mathrm{N}$ group, $\mathrm{H}$ group, $\mathrm{H}+\mathrm{DMSO}$ group (1:1,000 DMSO) and H + LY294002 group (20 $\mu \mathrm{mol} / 1$ LY294002). After $48 \mathrm{~h}$ at $37^{\circ} \mathrm{C}$, the related detections were performed.

shRNA plasmid construction. The pGenesil-1 plasmid was used to construct FBXW7 shRNA recombinant plasmid. A total of two single-stranded DNA oligonucleotides aimed at the FBXW7 gene were annealed to form double-stranded DNA, which was linked with pGenesil-1 digested with BamHI and HindIII using a T4 ligase at $16^{\circ} \mathrm{C}$ overnight. Then, the mixture was transfected into $E$. coil strain DH5 $\alpha$ for cloning and plasmid extraction. Finally, two constructs were designated pGenesil-1-FBXW7-1 and pGenesil-1-FBXW7-2 after sequencing identification. The pGenesil-1-FBXW7-1 targeted the sequence 5'-ACAGGACAG TGTTTACAAA-3', and pGenesil-1-FBXW7-2 targeted the sequence 5'-CAACAACGACGCCGAATTA-3'.

Plasmid transfection. Lipofectamine 2000 was used in HKC cells to perform transient transfection. HKC cells seeded on cover slides in 6-well plate were treated at $37^{\circ} \mathrm{C}$ for $5 \mathrm{~h}$ with $250 \mu \mathrm{l}$ serum-free DMEM (Gibco; Thermo Fisher Scientific, Inc.) containing $3.0 \mu \mathrm{g}$ plasmid and $10 \mu \mathrm{l}$ Lipofectamine 2000. After $5 \mathrm{~h}$, this medium was replaced with normal 
DMEM containing 10\% FBS (Biological Industries). At $48 \mathrm{~h}$ after transfection, the cells were collected for the subsequent experimentations.

Immunohistochemistry and immunocytochemistry. Renal cortex tissues were resected from the kidneys and fixed immediately in $4 \%$ paraformaldehyde for $48 \mathrm{~h}$ at room temperature. Subsequently, $4 \mu \mathrm{m}$ paraffin-embedded sections were made for immunohistochemistry. After deparaffinization (100\% xylene, room temperature, $30 \mathrm{~min}$ ), hydration (gradient ethanol series, room temperature, $10 \mathrm{~min}$ each), antigen recovery (citrate buffer $\mathrm{pH} 6.0,100^{\circ} \mathrm{C}, 5 \mathrm{~min}$ ) and blocking (5\% goat serum; cat. no. SP9000; OriGene Technologies, Inc.), $37^{\circ} \mathrm{C}, 20 \mathrm{~min}$ ), sections were incubated with primary antibodies against FBXW7 or SREBP-1 at $4^{\circ} \mathrm{C}$ overnight. The next morning sections were washed with PBS and then incubated with ready-to-use biotin-labelled secondary antibody (cat. no. SP9000; OriGene Technologies, Inc.) for $30 \mathrm{~min}$ at $37^{\circ} \mathrm{C}$. After incubation with horseradish peroxidase (HRP)-labelled streptavidin for $30 \mathrm{~min}$ at $37^{\circ} \mathrm{C}$, sections was visualized using DAB (OriGene Technologies, Inc.) for $10 \mathrm{sec}$ at room temperature. A negative control was established by replacing specific antibody with PBS. The sections were imaged with Olympus light microscope (Olympus Corporation) at $\mathrm{x} 400$ magnification.

Immunocytochemistry was used to investigate the expression levels of FBXW7 and SREBP-1 in HKC cells. After fixation (4\% paraformaldehyde, room temperature, $15 \mathrm{~min}$ ), permeabilization $\left(0.3 \%\right.$ Triton $\left.\mathrm{X}-100,37^{\circ} \mathrm{C}, 10 \mathrm{~min}\right)$ and blocking ( $5 \%$ goat serum, $\left.37^{\circ} \mathrm{C}, 30 \mathrm{~min}\right)$, cells were incubated with specific primary antibody of FBXW7 overnight at $4^{\circ} \mathrm{C}$. The following day, cells were incubated with biotin-labelled secondary antibody at $37^{\circ} \mathrm{C}$ for $30 \mathrm{~min}$ and HRP-labelled streptavidin at $37^{\circ} \mathrm{C}$ for $30 \mathrm{~min}$, in turn. Finally, cells were stained with DAB at room temperature for $20 \mathrm{sec}$, followed by image capture using light microscope at x400 magnification.

Immunofluorescence. After fixation using $4 \%$ paraformaldehyde at room temperature for $48 \mathrm{~h}, 4 \mu \mathrm{m}$ sections were made according to the standard procedure. After deparaffinization (100\% xylene, room temperature, $30 \mathrm{~min}$ ), hydration (gradient ethanol series, room temperature, $10 \mathrm{~min}$ each) and blocking (5\% goat serum, $\left.37^{\circ} \mathrm{C}, 20 \mathrm{~min}\right)$, tissue sections were co-incubated with primary antibodies of FBXW7 and SREBP- 1 at $4^{\circ} \mathrm{C}$ overnight. Then, after rinsing with PBS, sections were co-incubated with DyLight 488 labelled-goat anti-rabbit secondary antibody and DyLight 594 labelled-goat anti-mouse secondary antibody at $37^{\circ} \mathrm{C}$ for $30 \mathrm{~min}$. After counter-staining with DAPI at room temperature for $10 \mathrm{~min}$, positive signals were observed under fluorescence microscope and images were captured at $\mathrm{x} 400$ magnification.

For cultured cells, after fixation, permeabilization and blocking, cells were incubated with primary antibody against FBXW7 overnight at $4^{\circ} \mathrm{C}$ and DyLight 488 -labelled secondary antibody at $37^{\circ} \mathrm{C}$ for $2 \mathrm{~h}$, in turn. Positive signals were observed under fluorescence microscope at x400 magnification following DAPI staining at room temperature for $10 \mathrm{~min}$.

$R T$-quantitative ( $R T$-q)PCR. Total RNA was extracted from HKC cells using TRIzol reagent and reverse transcribed using
RT primer mix and RT enzyme mix (PrimeScript ${ }^{\mathrm{TM}}$ reverse transcription reagent kit with gDNA Eraser, Takara Bio, Inc.) at $37^{\circ} \mathrm{C}$ for $15 \mathrm{~min}$ after quantification. Equal amounts of RT reaction product $(2 \mu \mathrm{l})$ were subjected to PCR amplification and the SYBR-Green (Takara Bio, Inc.) qPCR method was used to determine FBXW7 mRNA expression. The thermocycling conditions were as follows: Initial denaturation for $3 \mathrm{~min}$ at $95^{\circ} \mathrm{C}$, followed by 40 cycles of denaturation at $95^{\circ} \mathrm{C}$ for $5 \mathrm{sec}$, annealing at $55^{\circ} \mathrm{C}$ for $30 \mathrm{sec}$ and extension at $72^{\circ} \mathrm{C}$ for $30 \mathrm{sec}$. The final extension was performed at $72^{\circ} \mathrm{C}$ for $10 \mathrm{~min}$. GAPDH was used as a housekeeping gene. The results were analyzed using the $2^{-\Delta \Delta C q}$ method (17). The primers sequences are presented in Table I.

Western blotting. Total protein extracted from HKC cells (RIPA lysis buffer; Beijing Solarbio Science \& Technology Co., Ltd.) was quantified using a Bio-Rad Quick Start Bradford Protein Assay kit (Bio-Rad Laboratories, Inc.). An equal amount of protein $(30 \mu \mathrm{g})$ was separated via $10 \%$ SDS-PAGE. Then, protein was transferred onto PVDF membranes (EMD Millipore), which were blocked with 5\% BSA (Biosharp Life Sciences) for $1 \mathrm{~h}$ at $37^{\circ} \mathrm{C}$. Next, blots were incubated with primary antibodies for FBXW7, SREBP-1 and $\beta$-actin overnight at $4^{\circ} \mathrm{C}$. The following morning, blots were washed with TBS-0.1\% Tween-20 (TBST) and incubated with HRP-conjugated secondary antibody for $2 \mathrm{~h}$ at room temperature. After rinsing with TBST, blots were incubated with ECL solution (Tiangen Biotech Co., Ltd.) for $2 \mathrm{~min}$ at room temperature and bands were visualized. For semi-quantitative analysis, bands were evaluated with LabWorks v4.5 software (Analytik Jena UVP) and normalized to $\beta$-actin density. The data are representative of three independent experiments.

Statistical analysis. Data are presented as the mean \pm SD and were analyzed with SPSS 13.0 for Windows (SPSS, Inc.). One-way ANOVA followed by Bonferroni post hoc test was used to determine significant differences among $>2$ groups. Unpaired Student's t-test was performed to determine the significance between two groups. $\mathrm{P}<0.05$ was considered to indicate a statistically significant difference. All experiments were independently repeated three times.

\section{Results}

FBXW7 and SREBP-1 expression levels in renal tubular cells of diabetic mice. FBXW7 and SREBP-1 expression levels were detected via immunohistochemistry in normal mice and diabetic mice. As presented in Fig. 1A, FBXW7 protein expression was located in the glomeruli and renal tubular cells of normal mice and diabetic mice. Compared with normal mice, diabetic mice had a lower expression of FBXW7 in renal tubular cells. On the contrary, SREBP-1 expression was increased in renal tubular cells of diabetic mice in comparison with normal mice. Furthermore, the potential co-expression of FBXW7 and SREBP-1 was analyzed using a double staining immunofluorescence technique, and it was identified that there was a definite co-expression of FBXW7 and SREBP-1 in renal tubular cells. Different to the immunohistochemistry findings, the results of immunofluorescence also identified the expression of SREBP-1 in the glomeruli, which may be due 
Table I. Primers of human SREBP-1 and GAPDH.

\begin{tabular}{lcc}
\hline Gene & Forward primer & Reverse primer \\
\hline Human SREBP-1 & 5'-AAAGAGTTGTTAGCGGTTCTCG-3' & 5'-CCACATGGATACCATCAAACTG-3' \\
Human GAPDH & 5'-GTCAACGGATTTGGTCGTATTG-3' & 5'-TGTAGTTGAGGTCAATGAAGGG-3' \\
\hline
\end{tabular}

SREBP-1, sterol regulatory element-binding protein 1 .

to the high sensitivity of immunofluorescence. However, there was no co-expression of FBXW7 and SREBP-1 in glomeruli, which suggested the diverse location of FBXW7 and SREBP-1 in renal glomeruli (Fig. 1B). The exact location of FBXW7 in glomeruli was further examine using double staining immunofluorescence with antibodies against FBXW7 and nestin (biomarker of podocyte). The partial co-expression of FBXW7 and nestin suggested that FBXW7 was also located in podocytes, besides renal tubular cells (Fig. S1).

High glucose decreases FBXW7 expression and increases SREBP-1 expression in HKC cells. Hyperglycemia is the most important characteristic of DM (18), and high glucose was used to treat $\mathrm{HKC}$ cells in the present study. It was identified that FBXW7 was decreased after treatment with high glucose for 36, 48 and $72 \mathrm{~h}$ compared with the corresponding $\mathrm{N}$ group (Fig. 2A). Statistical analysis demonstrated that FBXW7 expression was decreased by $70 \%$ in HKC cells treated with high glucose for $72 \mathrm{~h}$ compared with those cells treated with normal glucose. Moreover, there was no significant difference in the expression of FBXW7 between $\mathrm{N}$ group and $\mathrm{M}$ group at the indicated time points. Similarly, the results of immunofluorescence demonstrated that FBXW7 was located in the cytoplasm of HKC cells and was downregulated after treatment with high glucose in comparison with normal glucose treatment (Fig. 2B).

SREBP-1 expression was detected via western blotting, and the immunoblots were representative of three independent experiments. The precursor segment of SREBP-1 was increased by $3.75,4.16$ and 3.15 fold after treatment with high glucose for 36,48 and $72 \mathrm{~h}$, respectively, compared with the $\mathrm{N}$ group. Furthermore, the mature segment of SREBP-1 was enhanced by $2.67,4.27$ and 3.83 fold after treatment with high glucose (Fig. 2C).

Overexpression of FBXW7 decreases SREBP-1 expression in HKC cells. To elucidate the direct effect of FBXW7 on SREBP-1, FBXW7 expression was overexpression via transfection with pcDNA3.0-FBXW7 plasmid in HKC cells. The RT-qPCR results identified a 10.12-fold increase in FBXW7 mRNA expression (Fig. 3A). Furthermore, the immunocytochemistry demonstrated that the overexpression of FBXW7 appeared as increased brown staining in HKC cells transfected with pcDNA3.0-FBXW7, compared with that observed in cells transfected with pcDNA3.0. It was found that SREBP-1 expression was downregulated, as detected via immunocytochemistry (Fig. 3B). Overexpression of FBXW7 also led to smaller-sized HKC cells compared with those found in the pcDNA3.0 blank plasmid transfection group.
A
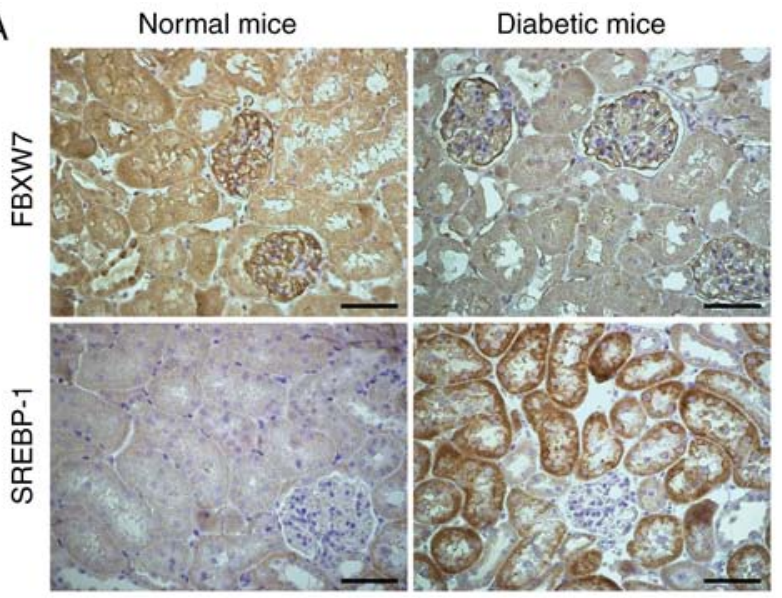

B
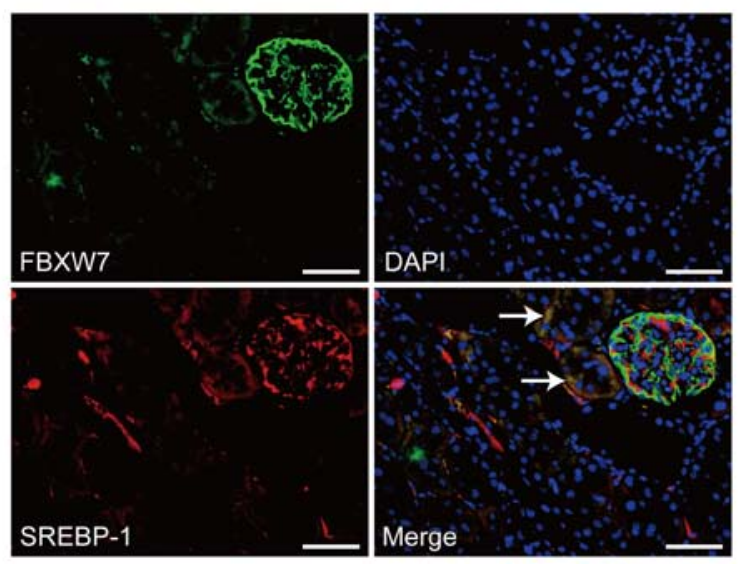

Figure 1. Expression levels of FBXW7 and SREBP-1 in the kidney of normal mice and diabetic mice. (A) Immunohistochemistry results of FBXW7 and SREBP-1 expression levels in the kidney of normal mice and diabetic mice. Scale bar, $50 \mu \mathrm{m}$. (B) Immunofluorescence double staining of FBXW7 and SREBP-1 expression levels in the kidney of normal mice and diabetic mice. Scale bar, $50 \mu \mathrm{m}$. White arrows indicate the co-expression of FBXW7 and SREBP-1. FBXW7, F-box and WD repeat domain containing 7; SREBP-1, sterol regulatory element-binding protein 1 .

Subsequently, western blotting was conducted and the results indicated that pcDNA3.0-FBXW7 plasmid transfection increased FBXW7 expression by 1.29-fold compared with the pcDNA3.0 transfection. Moreover, the precursor segment and mature segment of SREBP-1 were decreased by 11.11 and 2.63 fold, respectively, in HKC cells transfected with pcDNA3.0-FBXW7 plasmid compared with those transfected with pcDNA3.0 (Fig. 3C).

Downregulation of FBXW7 expression increases SREBP-1 expression in HKC cells. The effect of FBXW7 on SREBP-1 expression in HKC cells was further examined by knocking 
A
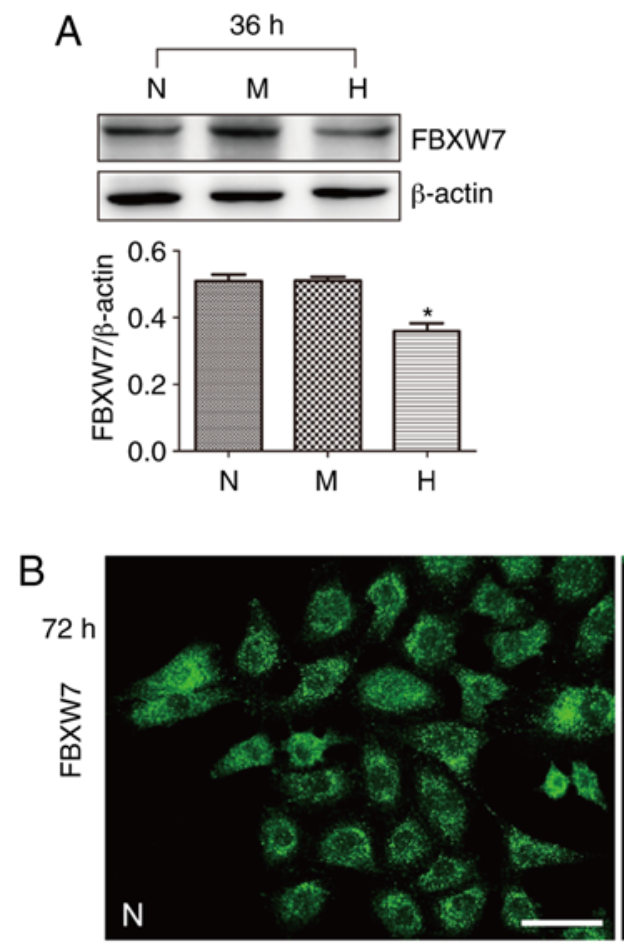

C
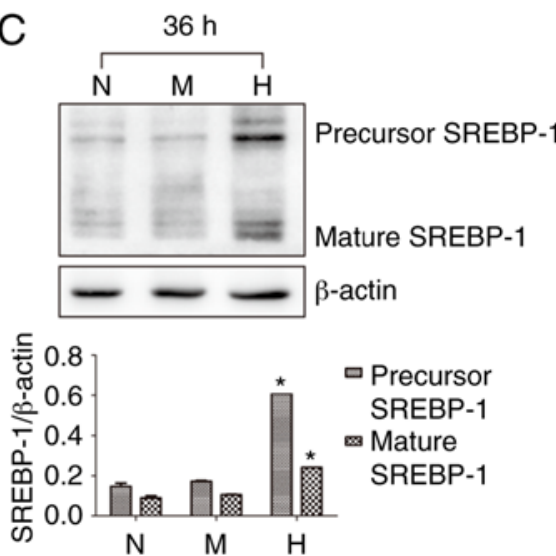
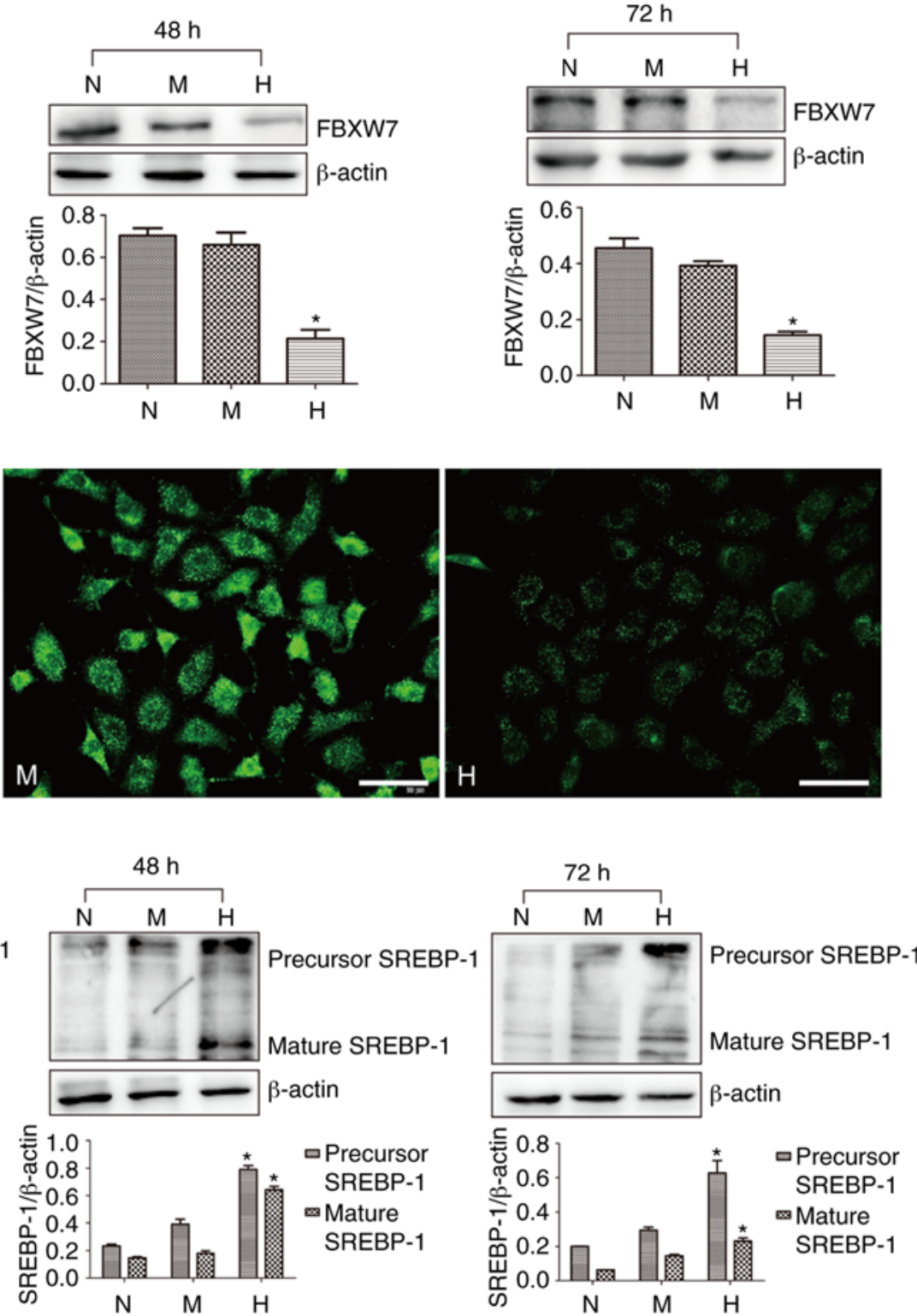

Figure 2. Expression levels of FBXW7 and SREBP-1 in H-cultured HKC cells. (A) Western blotting and statistical analysis of FBXW7 expression in HKC cells treated with N, H and M for 36, 48 and $72 \mathrm{~h}$. (B) Immunofluorescence of FBXW7 expression in HKC cells treated with N, H and M for 48 h. Scale bar, $50 \mu \mathrm{m}$. (C) Western blotting and statistical analysis of precursor segment of SREBP-1 and mature segment of SREBP-1 in HKC cells treated with N, H and M for 36, 48 and 72 h. "P<0.05 vs. N group. N, normal glucose; M, mannitol; FBXW7, F-box and WD repeat domain containing 7; SREBP-1, sterol regulatory element-binding protein 1.

down FBXW7 expression via transfection with shRNA plasmid. As presented in Fig. 4A, the transfection efficiency of pGenesil-1, pGenesil-1-FBXW7-1 and pGenesil-1-FBXW7-2 was $>75 \%$, as indicated by GFP expression. RT-qPCR results demonstrated that FBXW7 mRNA expression was effectively decreased in both pGenesil-1-FBXW7-1-transfected and pGenesil-1-FBXW7-2-transfected HKC cells compared with pGenesil-1-transfected cells. Statistical analysis identified a $54 \%$ and a $56 \%$ downregulation in FBXW7 mRNA expression in pGenesil-1-FBXW7-1-transfected cells and pGenesil-1-FBXW7-2-transfected cells, respectively (Fig. 4B). In line with $\mathrm{RT}$-qPCR results, immunocytochemistry analysis found that pGenesil-1-FBXW7-1 and pGenesil-1-FBXW7-2 downregulated FBXW7 protein expression (Fig. 4C). Conversely, SREBP-1 expression was markedly enhanced with the transfection of pGenesil-1-FBXW7-1 and pGenesil-1-FBXW7-2 in HKC cells (Fig. 4D). In addition, the western blotting results found a $29 \%$ and a $41 \%$ downregulation in FBXW7 expression after the transfection of pGenesil-1-FBXW7-1 and pGenesil-1-FBXW7-2 in cells, compared with that in pGenesil-1 in HKC cells. A 2.84 and a 2.54 fold-increase in precursor SREBP-1 and mature SREBP-1 expression levels was identified after pGenesil-1-FBXW7-1 transfection, as well as a 3.52 and a 3.64 fold-increase after pGenesil-1-FBXW7-2 transfection (Fig. 4E).

Inhibition of the PI3K/Akt pathway enhances FBXW7 expression and decreases SREBP-1 expression in HKC cells. PI3K/Akt signaling has been revealed to be the main cell signaling pathway that regulates cell function in renal tubular cells of DN, such as lipid metabolism, epithelial-mesenchymal transition and autophagy $(14,19)$. The results indicated that in 

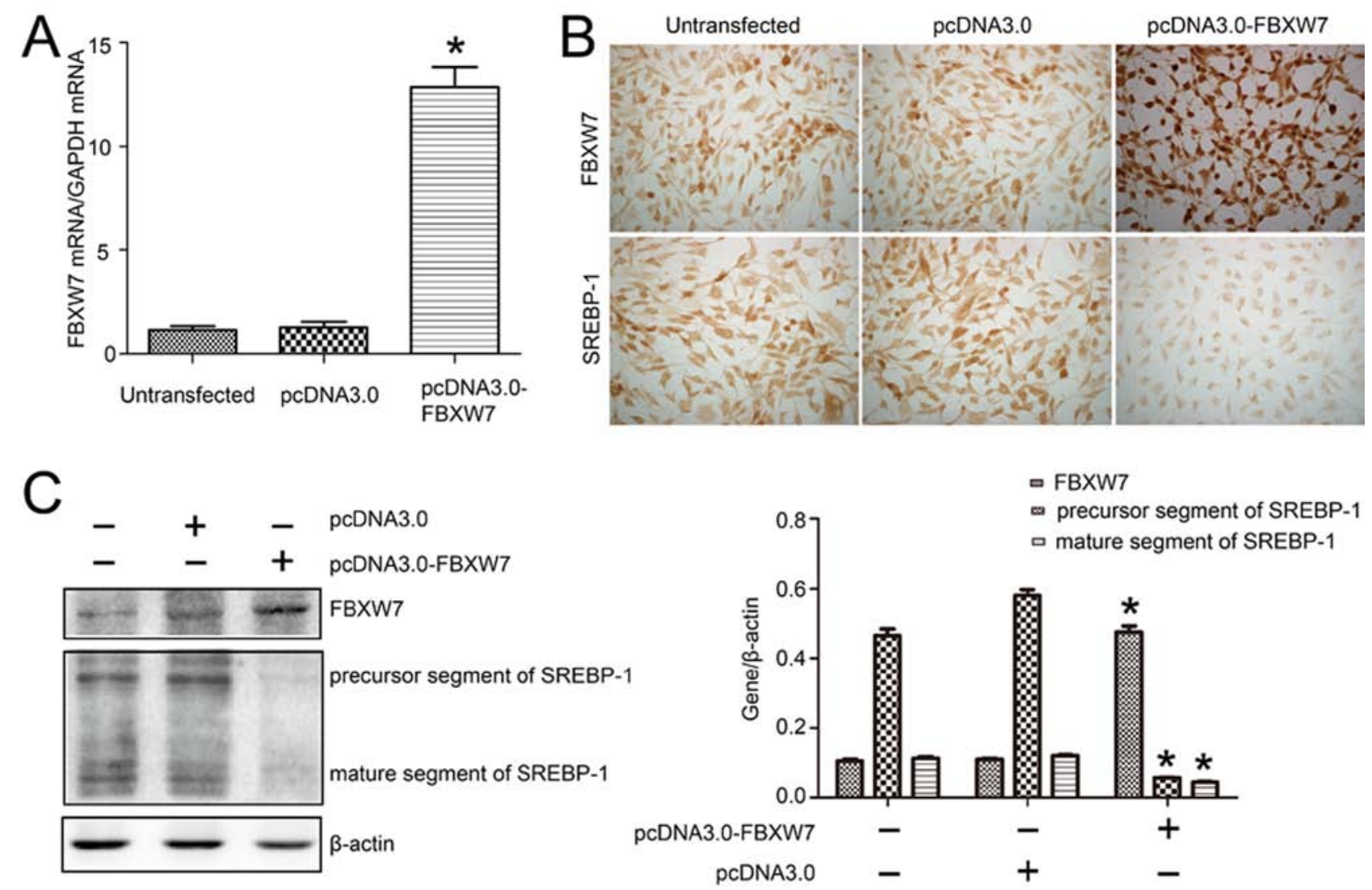

Figure 3. Overexpression of FBXW7 decreases SREBP-1 expression in HKC cells. (A) Reverse transcription-quantitative PCR results of FBXW7 mRNA expression in HKC cells of untransfected group, pcDNA3.0 group and pcDNA3.0-FBXW7 group. "P $<0.05$ vs. untransfected group. (B) Immunocytochemistry results of FBXW7 and SREBP-1 expression levels in HKC cells of untransfected group, pcDNA3.0 group and pcDNA3.0-FBXW7 group. Magnification, x400. (C) Western blotting and statistical analysis of FBXW7, precursor segment of SREBP-1 and mature segment of SREBP-1 expression levels in HKC cells of untransfected group, pcDNA3.0 group and pcDNA3.0-FBXW7 group. ${ }^{*} \mathrm{P}<0.05$ vs. pcDNA3.0 group. FBXW7, F-box and WD repeat domain containing 7; SREBP-1, sterol regulatory element-binding protein 1.

high glucose-cultured HKC cells, p-Akt (Ser 473)/Akt ratio was significantly increased compared with that in normal glucose-cultured HKC cells (Fig. 5A). Moreover, LY294002, a known PI3K/Akt pathway inhibitor, was used to determine the effect of the PI3K/Akt pathway on FBXW7 and SREBP-1 expression levels in HKC cells. It was found that FBXW7 expression was upregulated by 4.21 fold after LY294002 treatment compared with DMSO treatment (Fig. 5B). Furthermore, the precursor segment and mature segment of SREBP-1 were downregulated by 3.10 and 3.00 fold, respectively, by LY294002 treatment, compared with DMSO (Fig. 5B). Immunocytochemistry also demonstrated that LY294002 markedly decreased SREBP-1 expression, which presented as brown granules, in high glucose-cultured HKC cells (Fig. 5C).

\section{Discussion}

FBXW7 has been reported to be associated with the pathogenesis of DM. Brenachot et al (20) revealed that IL-1 $\beta$ and IFN- $\gamma$ downregulated FBXW7 expression in vitro insulin cells, followed by NF- $\kappa$ B pathway activation and cell apoptosis, which led to type I DM. Moreover, in type II DM, Zhao et al (21) observed that loss of FBXW7 in hepatic cells resulted in hyperglycemia, insulin resistance and impaired glucose tolerance. The present study identified the decreased expression of FBXW7 in renal tubular cells of DN. Similarly,
Tu et al (22) identified FBXW7 downregulation in the later stages of diabetic cardiomyopathy (>12 weeks). Therefore, these findings suggest that FBXW7 expression abnormality serves a role in the pathogenesis of DM and the corresponding complications.

The present study examined the potential regulatory factor of FBXW7 expression in renal tubular cells of DN using in vitro cultured cells. The results demonstrated that hyperglycemia was the main factor to inhibit FBXW7 expression in HKC cells. In line with the current findings, Gao et al (23) also reported that hyperglycemia was a regulatory factor of FBXW7 and could downregulate FBXW7 expression in renal mesangial cells, leading to diminished autophagy and increased inflammatory cytokines. Therefore, it was suggested that high glucose was the main factor to decrease FBXW7 and increase SREBP-1 expression levels in renal tubular cells of DN. Using double staining immunofluorescence in renal tissue it was also found that FBXW7 was located in podocytes, which indicated that, alongside renal tubular cells, the function of FBXW7 in podocytes requires further study.

The present study identified a negative relationship between FBXW7 and SREBP-1 expression in renal tubular cells of diabetic mice and in vitro HKC cells. This type of negative association between FBXW7 and SREBP-1 was also revealed in a study by Tu et al (24). These authors reported that in C57BL/6J mice fed with high fat diets the mRNA 


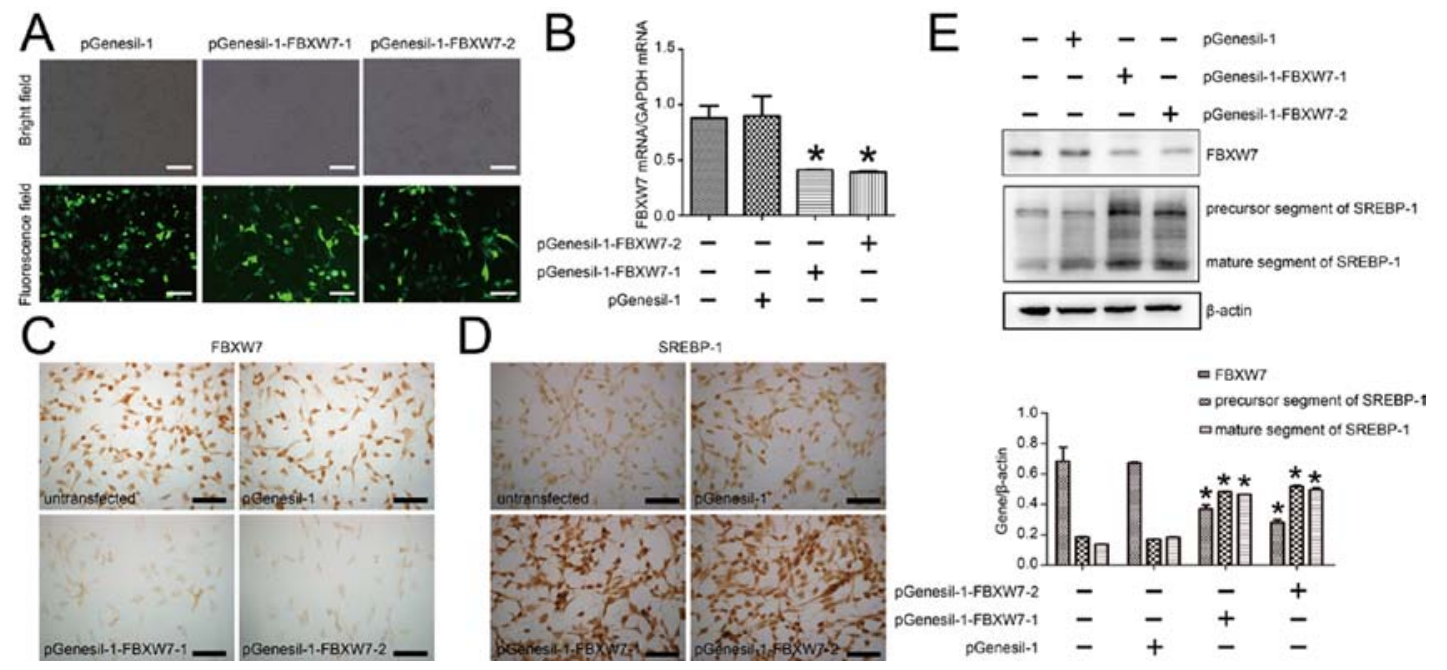

Figure 4. Knockdown of FBXW7 increases SREBP-1 expression in HKC cells. (A) Transfection efficiency of HKC cells transfected with pGenesil-1, pGenesil-1-FBXW7-1 and pGenesil-1-FBXW7-2. Scale bar, $100 \mu \mathrm{m}$. (B) Reverse transcription-quantitative PCR results of FBXW7 mRNA expression in HKC cells of untransfected group, pGenesil-1 group, pGenesil-1-FBXW7-1 group and pGenesil-1-FBXW7-2 group. (C) Immunocytochemistry results of FBXW7 expression in HKC cells of untransfected group, pGenesil-1 group, pGenesil-1-FBXW7-1 group and pGenesil-1-FBXW7-2 group. Scale bar, $50 \mu \mathrm{m}$. (D) Immunocytochemistry results of SREBP-1 expression in HKC cells of untransfected group, pGenesil-1 group, pGenesil-1-FBXW7-1 group and pGenesil-1-FBXW7-2 group. Scale bar, $50 \mu \mathrm{m}$. (E) Western blotting and statistical analysis of FBXW7, precursor segment of SREBP-1 and mature segment of SREBP-1 expression levels in HKC cells of untransfected group, pGenesil-1 group, pGenesil-1-FBXW7-1 group and pGenesil-1-FBXW7-2 group. "P<0.05 vs. pGenesil-1 group. FBXW7, F-box and WD repeat domain containing 7; SREBP-1, sterol regulatory element-binding protein 1.
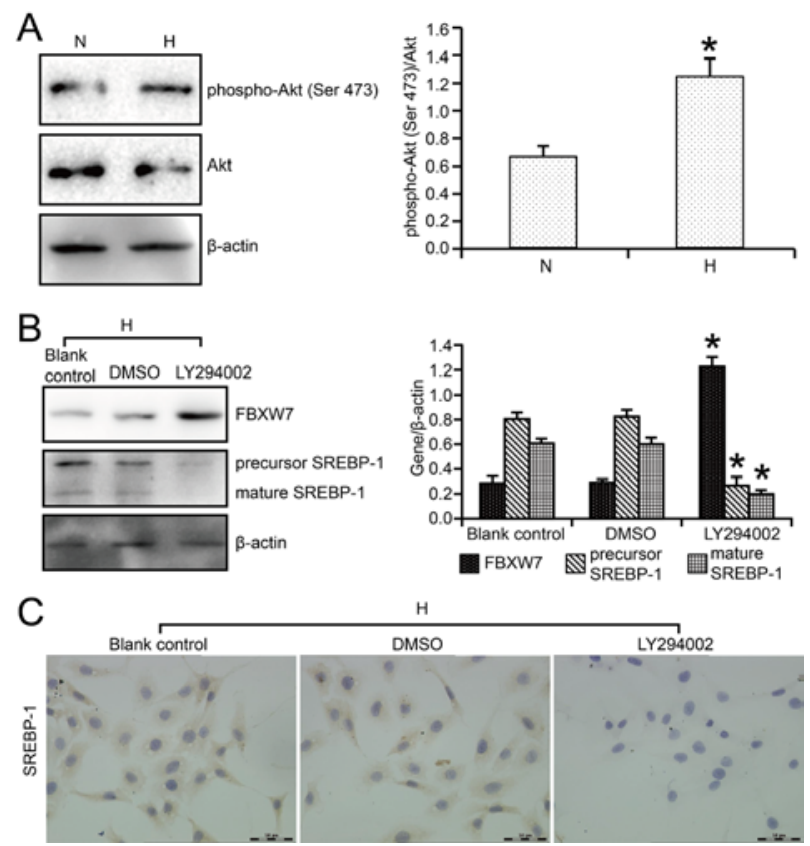

Figure 5. Inhibition of the PI3K/Akt pathway reverses H-induced FBXW7 downregulation and SREBP-1 upregulation in HKC cells. (A) Western blotting of the effect of $\mathrm{H}$ on p-Akt (Ser 473) and Akt in HKC cells. "P<0.05 vs. $\mathrm{N}$ group. (B) Western blotting detection and statistical analysis the effect of LY294002 on FBXW7, precursor segment of SREBP-1 and mature segment of SREBP-1 expression levels in H-treated HKC cells. " $\mathrm{P}<0.05$ vs. DMSO group. (C) Immunocytochemistry results of SREBP-1 in H-treated HKC cells of blank control group, DMSO group and LY294002 group. Scale bar, $50 \mu \mathrm{m}$. $\mathrm{N}$ group. $\mathrm{N}$, normal glucose; $\mathrm{M}$, mannitol; FBXW7, F-box and WD repeat domain containing 7; SREBP-1, sterol regulatory element-binding protein 1 p-, phosphorylated.

and protein expression levels of Fbxw7 were significantly decreased in liver tissues, compared with normal diets group, which was negatively correlated with SREBP-1, indicating the role of the FBXW7/SREBP-1 axis in the development of non-alcoholic fatty liver disease (24). Moreover, the present study observed the co-location of FBXW7 and SREBP-1 in renal tubular cells of diabetic mice, but not in glomeruli, using immunofluorescence double staining. In line with this finding, our previous study revealed that SREBP-1 was mainly expressed in renal tubular cells, which could mediate lipid metabolism abnormality and ECM accumulation in DN $(9,25)$. However, immunofluorescence detection demonstrated the positive expression of SREBP-1 in glomeruli, while immunohistochemistry did not detect a positive signal of SREBP-1 in glomeruli. It was suggested that the sensitivity difference of the experimental methods may be the main cause of this discrepancy. Therefore, considering the negative relationship and co-expression of FBXW7 and SREBP-1, it was suggested that FBXW7 may negatively regulate SREBP-1 expression in renal tubular cells of DN.

To elucidate the direct regulation of FBXW7 on SREBP-1 in HKC cells, the present study overexpressed FBXW7, and subsequently found that SREBP-1 was downregulated. However, when FBXW7 was knocked down by RNA interference, SREBP-1 was upregulated. As an E3 ubiquitin ligase, FBXW7 has been reported to enhance SREBP-1a, SREBP-1c and SREBP-2 ubiquitination and degradation in a manner dependent on the phosphorylation of SREBPs, which is regulated by GSK-3 (26). Moreover inactivation of FBXW7 results in the stabilization of SREBPs, enhancing the synthesis of cholesterol and fatty acids via SREBPs downstream genes $(26,27)$. However, in a previous study, FBXW7 was also revealed to be regulated by SREBP-2 via microRNAs (miR), miR-182 and miR-96, and accumulating miR-182 and miR-96 negatively regulated FBXW7 and insulin induced gene- 2 expression levels (28). Thus, there may be a regulatory loop between FBXW7 and SREBPs. Therefore, along with the present data, it was suggested that FBXW7 
may be the upstream regulator of SREBP-1 in renal tubular cells of DN. Furthermore, the present results indicated that overexpression of FBXW7 caused smaller-sized HKC cells. Based on a previous study (29), it was suggested that FBXW7 upregulation may affect cell proliferation via its target c-Myc, as well as lipid metabolism via SREBP-1.

The PI3K/Akt pathway is a multifunctional signaling pathway and serves an important role in the pathogenesis and development of DN (30). In our previous study, the PI3K/Akt pathway was revealed to regulate SREBP-1 expression in renal tubular cells of DN (14). Furthermore, downstream proteins of Akt, GSK-3 $\beta$ and mTOR were found to be involved in PI3K/Akt pathway-regulated SREBP-1 expression and lipid metabolism in renal tubular cells of DN $(31,32)$. The present study demonstrated that PI3K/Akt pathway activation also regulated FBXW7 expression in HKC cells. LY294002, a known PI3K/Akt pathway inhibitor, improved the high glucose-induced decline in FBXW7 expression. Similarly, Suryo Rahmanto et al (33) reported that in medulloblastoma cells, pharmacological inhibition of the PI3K/Akt pathway enhanced FBXW7 expression and destabilized SOX9, rendering cells sensitive to cisplatin treatment. Therefore, the PI3K/Akt signaling pathway may be the upstream pathway that regulates FBXW7 expression in renal tubular cells of DN. However, to further elucidate the function and regulation of FBXW7 in DM, additional in vivo experiments using lentivirus containing FBXW7 CDS (coding sequence) or shRNA targeted at FBXW7 are required.

In conclusion, the present findings demonstrated that high glucose caused FBXW7 downregulation in renal tubular cells of diabetic mice, especially at late stage, and was negatively associated with SREBP-1 expression. Moreover, FBXW7 may be the upstream regulator of SREBP-1 in renal tubular cells of $\mathrm{DN}$, and it was found that the PI3K/Akt pathway mediated the high glucose-induced downregulation of FBXW7 expression in renal tubular cells. Therefore, combined therapies targeted at both FBXW7 and SREBP-1 may be an effective strategy to prevent $\mathrm{DN}$ by regulating lipid metabolism.

\section{Acknowledgements}

The authors would like to thank Professor Huachuan Zheng (Department of Experimental Oncology and Animal Center, Shengjing Hospital of China Medical University) for helpful guidance in biotechnology.

\section{Funding}

This work was supported by a grant from the Natural Science Foundation of Hebei Province (grant no. H2018206096).

\section{Availability of data and materials}

The datasets used and/or analyzed during the current study are available from the corresponding author on reasonable request.

\section{Authors' contributions}

JH and LZ designed the research. LL, JY, FL and FG conducted the experiment. LL, LZ and JH analyzed the data. $\mathrm{LZ}$ and $\mathrm{JH}$ wrote the manuscript. All authors read and approved the final manuscript.

\section{Ethics approval and consent to participate}

All mice were treated according to the guidelines of Institutional Animal Care and Use Committee of the Third Hospital of Hebei Medical University.

\section{Patient consent for publication}

Not applicable.

\section{Competing interests}

The authors declare that they have no competing interests.

\section{References}

1. Ramzy MM, Abdalla AM, Zenhom NM, Okasha AM, Abdelkafy AE and Saleh RK: Therapeutic effect of liraglutide on expression of CTGF and BMP-7 in induced diabetic nephropathy. J Cell Biochem 120: 17512-17519, 2019.

2. Yaribeygi H, Mohammadi MT, Rezaee R and Sahebkar A: Fenofibrate improves renal function by amelioration of NOX-4, IL-18, and p53 expression in an experimental model of diabetic nephropathy. J Cell Biochem 119: 7458-7469, 2018.

3. Forbes JM and Thorburn DR: Mitochondrial dysfunction in diabetic kidney disease. Nat RevNephrol 14: 291-312, 2018.

4. Cybulsky AV: Endoplasmic reticulum stress, the unfolded protein response and autophagy in kidney diseases. Nat Rev Nephrol 13: 681-696, 2017.

5. Yang W, Luo Y, Yang S, Zeng M, Zhang S, Liu J, Han Y, Liu Y, Zhu X, Wu H, et al: Ectopic lipid accumulation: Potential role in tubular injury and inflammation in diabetic kidney disease. Clin Sci (Lond) 132: 2407-2422, 2018.

6. Fornoni A, Merscher S and Kopp JB: Lipid biology of the podocyte-new perspectives offer new opportunities. Nat Rev Nephrol 10: 379-388, 2014.

7. Brosius FC III: New insights into the mechanisms of fibrosis and sclerosis in diabetic nephropathy. Rev Endocr Metab Disord 9: 245-254, 2008.

8. Soyal SM, Nofziger C, Dossena S, Paulmichl M and Patsch W: Targeting SREBPs for treatment of the metabolic syndrome. Trends Pharmacol Sci 36: 406-416, 2015.

9. Jun H, Song Z, Chen W, Zanhua R, Yonghong S, Shuxia L and Huijun D: In vivo and in vitro effects of SREBP-1 on diabetic renal tubular lipid accumulation and RNAi-mediated gene silencing study. Histochem Cell Biol 131: 327-345, 2009.

10. Zhou Z, He C and Wang J: Regulation mechanism of Fbxw7-related signaling pathways (Review). Oncol Rep 34: 2215-2224, 2015.

11. Kourtis N, Strikoudis A and Aifantis I: Emerging roles for the FBXW7 ubiquitin ligase in leukemia and beyond. Curr Opin Cell Biol 37: 28-34, 2015.

12. Yumimoto $\mathrm{K}$ and Nakayama KI: Recent insight into the role of FBXW7 as a tumor suppressor. Semin Cancer Biol 67: 1-15, 2020.

13. Fiore D, Piscopo C, Proto MC, Vasaturo M, Piaz FD, Fusco BM, Pagano C, Laezza C, Bifulco M and Gazzerro P: N6-Isopentenyladenosine inhibits colorectal cancer and improves sensitivity to 5-fluorouracil-targeting FBXW7 tumor suppressor. Cancers (Basel) 28: 1456, 2019.

14. Hao J, Liu S, Zhao S, Liu Q, Lv X, Chen H, Niu Y and Duan H: PI3K/Akt pathway mediates high glucose-induced lipogenesis and extracellular matrix accumulation in HKC cells through regulation of SREBP-1 and TGF- $\beta 1$. Histochem Cell Biol 135: 173-181, 2011.

15. Du W, Wang N, Li F, Jia K, An J, Liu Y, Wang Y, Zhu L, Zhao S and Hao J: STAT3 phosphorylation mediates high glucose-impaired cell autophagy in an HDAC1-dependent and -independent manner in schwann cells of diabetic peripheral neuropathy. FASEB J 33: 8008-8021, 2019. 
16. Racusen LC, Monteil C, Sgrignoli A, Lucskay M, Marouillat S, Rhim JG and Morin JP: Cell lines with extended in vitro growth potential from human renal proximal tubule: Characterization, response to inducers, and comparison with established cell lines. J Labo Clin Med 129: 318-329, 1997.

17. Livak KJ and Schmittgen TD: Analysis of relative gene expression data using real-time quantitative PCR and the 2(-Delta Delta C(T)) method. Methods 25: 402-408, 2001.

18. Ceriello A: Postprandial hyperglycemia and diabetes complications: Is it time to treat? Diabetes 541: 1-7, 2005

19. Zhuang L, Jin G, Hu X, Yang Q and Shi Z: The inhibition of SGK1 suppresses epithelial-mesenchymal transition and promotes renal tubular epithelial cell autophagy in diabetic nephropathy. Am J Transl Res 118: 4946-4956, 2019.

20. Brenachot X, Ramadori G, Ioris RM, Veyrat-Durebex C, Altirriba J, Aras E, Ljubicic S, Kohno D, Fabbiano S, Clement S, et al: Hepatic protein tyrosine phosphatase receptor gamma links obesity-induced inflammation to insulin resistance. Nat Commun 8: 1820, 2017.

21. Zhao J, Xiong X, Li Y, Liu X, Wang T, Zhang H, Jiao Y, Jiang J, Zhang H, Tang Q, et al: Hepatic F-box protein FBXW7 maintains glucose homeostasis through degradation of fetuin-A. Diabetes 67: 818-830, 2018

22. Tu Lu-Mei LM, Wang Y and Yan-Ling MU: Expression of FBXW7 in diabetic cardiomyopathy. Chin J Pathophysiol 34: 2271-2276, 2018.

23. Gao C, Fan F, Chen J, Long Y, Tang S, Jiang C and Xu Y: FBW7 regulates the autophagy signal in mesangial cells induced by high glucose. Biomed Res Int 2019: 6061594, 2019.

24. Tu K, Zheng X, Yin G, Zan X, Yao Y and Liu Q: Evaluation of Fbxw7 expression and its correlation with expression of SREBP-1 in a mouse model of NAFLD. Mol Med Rep 6: 525-530, 2012.

25. Hao J, Zhu L, Zhao S, Liu S, Liu Q and Duan H: PTEN ameliorates high glucose-induced lipid deposits through regulating SREBP-1/FASN/ACC pathway in renal proximal tubular cells. Exp Cell Res 317: 1629-1639, 2011.

26. Sundqvist A, Bengoechea-Alonso MT, Ye X, Lukiyanchuk V, Jin J, Harper JW and Ericsson J: Control of lipid metabolism by phosphorylation-dependent degradation of the SREBP family of transcription factors by SCF(Fbw7). Cell Metab 1: 379-391, 2005.
27. Bengoechea-Alonso MT and Ericsson J: The phosphorylation-dependent regulation of nuclear SREBP1 during mitosis links lipid metabolism and cell growth. Cell Cycle 15: 2753-2765, 2016.

28. Jeon TI, Esquejo RM, Roqueta-Rivera M, Phelan PE, Moon YA, Govindarajan SS, Esau CC and Osborne TF: An SREBP-responsive microRNA operon contributes to a regulatory loop for intracellular lipid homeostasis. Cell Metab 18: 51-61, 2013.

29. Yeh $\mathrm{CH}$, Bellon $\mathrm{M}$ and Nicot C: FBXW7: a critical tumor suppressor of human cancers. Mol Cancer 17: 115, 2018.

30. Lei S, Su W, Xia ZY, Wang Y, Zhou L, Qiao S, Zhao B, , Xia Z, Irwin M: Hyperglycemia-Induced Oxidative Stress Abrogates Remifentanil Preconditioning-Mediated Cardioprotection in Diabetic Rats by Impairing Caveolin-3-Modulated PI3K/Akt and JAK2/STAT3 Signaling. Oxid Med Cell Longev 2019: 9836302, 2019.

31. Hao J, Zhu L, Li F, Liu Q, Zhao X, Liu S, Xing L, Feng X and Duan H: Phospho-mTOR: A novel target in regulation of renal lipid metabolism abnormality of diabetes. Exp Cell Res 319: 2296-2306, 2013.

32. Liu W, Hao J, Zhu L, Li F, Liu Q, Liu S, Zhao S, Li H and Duan H: Phospho-GSK-3 $\beta$ is involved in the high-glucose-mediated lipid deposition in renal tubular cells in diabetes. Int J Biochem Cell Biol 45: 2066-2075, 2013.

33. Suryo Rahmanto A, Savov V, Brunner A, Bolin S, Weishaupt H, Malyukova A, Rosén G, Čančer M, Hutter S and Sundström A: FBW7 suppression leads to SOX9 stabilization and increased malignancy in medulloblastoma. EMBO J 35: 2192-2212, 2016.

This work is licensed under a Creative Commons Attribution-NonCommercial-NoDerivatives 4.0 International (CC BY-NC-ND 4.0) License. 